\title{
Derivation of a merging condition for two interacting streamers in air
}

\author{
Zdeněk Bonaventura \\ Department of Physical Electronics, \\ Faculty of Science, Masaryk University, \\ Kotlářská 2, 61137 Brno, Czech Republic \\ zbona@physics.muni.cz \\ Max Duarte \\ Laboratoire J.-A. Dieudonné - UMR CNRS 6621, \\ Université de Nice - Sophia Antipolis, \\ Parc Valrose, 06108 Nice Cedex 02, France
}

Anne Bourdon

CNRS, UPR 288 Laboratoire EM2C, Grande voie des vignes, 92295 Châtenay-Malabry, France

Ecole Centrale Paris,

Grande voie des vignes, 92295 Châtenay-Malabry, France

Marc Massot

CNRS, UPR 288 Laboratoire EM2C, Grande voie des vignes, 92295 Châtenay-Malabry, France

Ecole Centrale Paris,

Grande voie des vignes, 92295 Châtenay-Malabry, France

Center for Turbulence Research, Stanford University, Building 500, 488 Escondido Mall, Stanford CA 94305-3035, USA

October 17, 2018

\begin{abstract}
The simulation of the interaction of two simultaneously propagating air streamers of the same polarity is presented. A parametric study has been carried out using an accurate numerical method which ensures a time-space error control of the solution. For initial separation of both streamers smaller or comparable to the longest characteristic absorption length of photoionization in air, we have found that the streamers tend to merge at the moment when the ratio between their characteristic width and their mutual distance reaches a value of about 0.35 for positive streamers, and 0.4 for negative ones. Moreover it is demonstrated that these ratios are practically independent of the applied electric field, the initial seed configuration, and the pressure.
\end{abstract}

Electrical breakdown in air gaps often involves the development of fast ionizing waves that take the form of thin filaments called streamers [1. These complex and highly nonlinear phenomena precede the formation of sparks and leaders. Similar filamentary structures 
are observed in sprites: large-scale discharges which appear at low pressure in the altitude range of about 40 to $90 \mathrm{~km}$ above large thunderstorms [2, 3]. Streamers often form branches. Then the individual streamer heads carry charges of the same polarity, and thus they are electrostatically repelling each other. However, careful observations show also the opposite behavior: streamers may even merge or reconnect [4, 5, 6. So far, only few numerical studies have been carried out on streamer interactions. First, the impact of charges of numerous positive streamers, propagating simultaneously, on the electric field and on the velocity of one single positive streamer was studied in [7] for a 2D configuration. Later on it was shown in 8 . that the electrodynamics of a 2D array of negative streamers substantially differs from the one of a single streamer due to their electrostatic interaction. Recently, the interaction of two streamer discharges in air and other oxygen-nitrogen mixtures has been studied for a 3D cylindrical configuration in [9. These authors showed that two competing mechanisms have to be considered: attraction due to nonlocal photoionization between streamers and electrostatic repulsion of streamer heads due to their space charges of same polarity. Qualitatively, they concluded that streamer merging is favored if the distance between streamers is smaller or comparable to the longest absorption length of photoionization. However, a more precise or even quantitative description of streamer merging remains an open problem.

In this work we carry out a parametric analysis of the dynamics of interacting streamers to better understand the conditions of streamer merging. Therefore we simulate the interaction of two streamers of the same polarity by means of an accurate numerical method which ensures a time-space error control of the solution. Taking into account that fully 3D simulations are still computationally expensive to carry out an extensive parametric analysis, we have considered a Cartesian 2D geometry to reduce computational costs. This study is then a first step towards a quantitative analysis of streamer merging in real 3D configurations. However, in agreement with [8, 10, we consider that the main characteristics of the dynamics of interacting streamers in two dimensions, as described below, will be similar in three dimensions. In practice, we aim at defining geometrical parameters independent of the multivariable complex physical settings and thus a simple characterization that may be extended to more general configurations.

We consider the classical fluid model for air given by drift-diffusion equations self-consistently coupled with Poisson's equation [11, 12]:

$$
\left.\begin{array}{c}
\partial_{t} n_{\mathrm{e}}+\nabla \cdot\left(n_{\mathrm{e}} \mathbf{v}_{\mathrm{e}}\right)-\nabla \cdot\left(D_{\mathrm{e}} \nabla n_{\mathrm{e}}\right)=n_{\mathrm{e}} \alpha\left|\mathbf{v}_{\mathrm{e}}\right|-n_{\mathrm{e}} \eta\left|\mathbf{v}_{\mathrm{e}}\right|-n_{\mathrm{e}} n_{\mathrm{p}} \beta_{\mathrm{ep}}+n_{\mathrm{n}} \gamma+S_{\mathrm{ph}}, \\
\partial_{t} n_{\mathrm{p}}+\nabla \cdot\left(n_{\mathrm{p}} \mathbf{v}_{\mathrm{p}}\right)-\nabla \cdot\left(D_{\mathrm{p}} \nabla n_{\mathrm{p}}\right)=n_{\mathrm{e}} \alpha\left|\mathbf{v}_{\mathrm{e}}\right|-n_{\mathrm{e}} n_{\mathrm{p}} \beta_{\mathrm{ep}}-n_{\mathrm{n}} n_{\mathrm{p}} \beta_{\mathrm{np}}+S_{\mathrm{ph}}, \\
\partial_{t} n_{\mathrm{n}}+\nabla \cdot\left(n_{\mathrm{n}} \mathbf{v}_{\mathrm{n}}\right)-\nabla \cdot\left(D_{\mathrm{n}} \nabla n_{\mathrm{n}}\right)=n_{\mathrm{e}} \eta\left|\mathbf{v}_{\mathrm{e}}\right|-n_{\mathrm{n}} n_{\mathrm{p}} \beta_{\mathrm{np}}-n_{\mathrm{n}} \gamma, \\
\varepsilon_{0} \nabla \cdot \mathbf{E}=-q_{\mathrm{e}}\left(n_{\mathrm{p}}-n_{\mathrm{n}}-n_{\mathrm{e}}\right), \quad \mathbf{E}=-\nabla V,
\end{array}\right\}
$$

where $n_{i}$ is the density of charged species $i$ (e: electrons, p: positive ions, n: negative ions), $V$ and $\mathbf{E}$ stand, respectively, for the electric potential and field, and $\mathbf{v}_{i}=\mu_{i} \mathbf{E}$ is the drift velocity. We denote by $D_{i}$ and $\mu_{i}$ the diffusion coefficient and the mobility of charged species $i, q_{\mathrm{e}}$ is the absolute value of the electron charge, and $\varepsilon_{0}$ is the permittivity of free space. Moreover $\alpha$ is the impact ionization coefficient, $\eta$ stands for the electron attachment coefficient, $\beta_{\mathrm{ep}}$ and $\beta_{\mathrm{np}}$ are, respectively, the electron-positive ion and negative-positive ion recombination coefficients, and $\gamma$ is the detachment coefficient. All these coefficients depend on the local reduced electric field $E / N$, and thus vary in time and space, where $E=|\mathbf{E}|$ is the electric field magnitude, and $N$ is the air neutral density. Further details are given in [15. For $S_{\mathrm{ph}}$, the photoionization source term for air, we use the 3-Group $\mathrm{SP}_{3}$ model derived in [13] with Larsen's boundary conditions [14. In this model, three equivalent absorption lengths are used to model the wavelength dependence of the photoionization source term in air. At ground pressure these absorption lengths are $\left(\lambda_{1} p_{\mathrm{O}_{2}}\right)^{-1}=0.1408 \mathrm{~cm},\left(\lambda_{2} p_{\mathrm{O}_{2}}\right)^{-1}=0.0561 \mathrm{~cm}$, and 
$\left(\lambda_{3} p_{\mathrm{O}_{2}}\right)^{-1}=0.0105 \mathrm{~cm}$, where $p_{\mathrm{O}_{2}}=158.9$ Torr is the partial pressure of molecular oxygen in air.

To handle the stiff modeling equations, we have recently developed in [15] a numerical scheme for multi-scale streamer discharge simulations for general Cartesian multi-dimensional geometries. The strategy is based on a second order time adaptive integration with a splitting technique and dedicated solvers, and space finite volume multiresolution for dynamic grid adaptation. These features involve important efficiency gains in terms of CPU time and memory space while ensuring a time-space error control of the solution. Poisson's equation and Helmholtz equations issued by the photoionization model are considered on the adapted grid, and the resulting sparse linear systems are solved with MUMPS [16, 17]. In all simulations carried out in this work (except for some computations in figure 6 where a finer grid is used) a time-space accuracy tolerance of $10^{-4}$ is used for a space resolution of $3.9 \mu \mathrm{m}$. This choice guarantees a sufficiently fine time-space representation of the physics, and numerical results disclosing practically the same physical behavior for higher spatial resolutions and tighter accuracy tolerances. The domain size is carefully chosen for the various configurations and with the same space resolution of $3.9 \mu \mathrm{m}$ such that no interference of the boundaries is evidenced.

In order to preserve similarity constraints at different pressures (corresponding to discharges at different altitudes), all variables are scaled by an appropriate power of the ratio of the air density at ground pressure $N_{0}$, and the air density $N$ at the given pressure [18. For example, the space dimensions scale as $\mathbf{x}=\mathbf{x}_{0} N_{0} / N$, the charge densities as $n=n_{0} N^{2} / N_{0}^{2}$, the electric field as $E=E_{0} N / N_{0}$, and the time as $t=t_{0} N_{0} / N$, where the subscript " 0 " refers to the values at ground pressure. Our reference (ground) density for air is $N_{0}=2.688 \times 10^{19} \mathrm{~cm}^{-3}$ at a temperature of $273 \mathrm{~K}$.

We study two separate discharge filaments initiated by placing two identical Gaussian plasma clouds into a homogeneous background electric field generated by two remote electrodes. The pressure, streamer polarity, applied electric field, and the configuration parameters of the initial Gaussian seeds (the width $\sigma$, the maximum density $n_{\max }$, and the mutual separation $y_{0}$ ) are varied in order to derive a condition for the merging of both streamers.

Figures 1(a)-(c) show snapshots of different phases of the evolution of the net charge density $\left(\rho=n_{\mathrm{p}}-n_{\mathrm{e}}-n_{\mathrm{n}}\right)$ for two simultaneously propagating positive discharge filaments at ground pressure $\left(N / N_{0}=1.0\right)$ for an initial seed separation of $y_{0}=0.2 N_{0} / N \mathrm{~cm}$, a maximum density of $n_{\max }=10^{13} N^{2} / N_{0}^{2} \mathrm{~cm}^{-3}$, a width $\sigma=0.02 N_{0} / N \mathrm{~cm}$, and an applied electric field $E_{\mathrm{a}}=1.5 E_{\mathrm{bd}}$, where $E_{\mathrm{bd}}=32 N / N_{0} \mathrm{kV} \mathrm{cm}^{-1}$ is the breakdown electric field in air. The complete trajectory of the maximum electric field is also shown. Following this trajectory, the transition time $t_{\mathrm{tr}}$, between the streamer repulsion and the beginning of their merging, is defined as the instant when the separation of the two trajectories starts to decrease. For the condition of Figure 1 we have $t_{\mathrm{tr}}=6.3 \mathrm{~ns}$. Figure 2(a) shows at this time the distribution of the net charge density. We define in Figure 2 (b) the mutual distance of both filaments $\delta$ by the separation of the maximum net density $\rho_{\max }$ in each head; and the streamer head characteristic width $\mathcal{D}$ as the planar length set by the cross section in the direction perpendicular to the applied electric field $\mathbf{E}_{\mathrm{a}}$, where the charge density is locally higher than half the maximum net density: $\rho \geq \rho_{\max } / 2$.

We have found that the merging condition for two streamers in air could be represented by the value of the ratio $\mathcal{D} / \delta$ which appears to be very stable for a given polarity of discharges. To illustrate this, Figure 3 shows the time evolution of the ratio $\mathcal{D} / \delta$ for two positive or two negative streamers. In this figure, initial separation of the seeds $y_{0}=0.15 N_{0} / N \mathrm{~cm}$ and $0.2 N_{0} / N \mathrm{~cm}$ are considered, and other parameters are similar to Figure 1. First, Figure 3 shows the time evolution of the ratio $\mathcal{D} / \delta$ for two negative streamers as these discharges may propagate without photoionization source term. When photoionization is included, the 


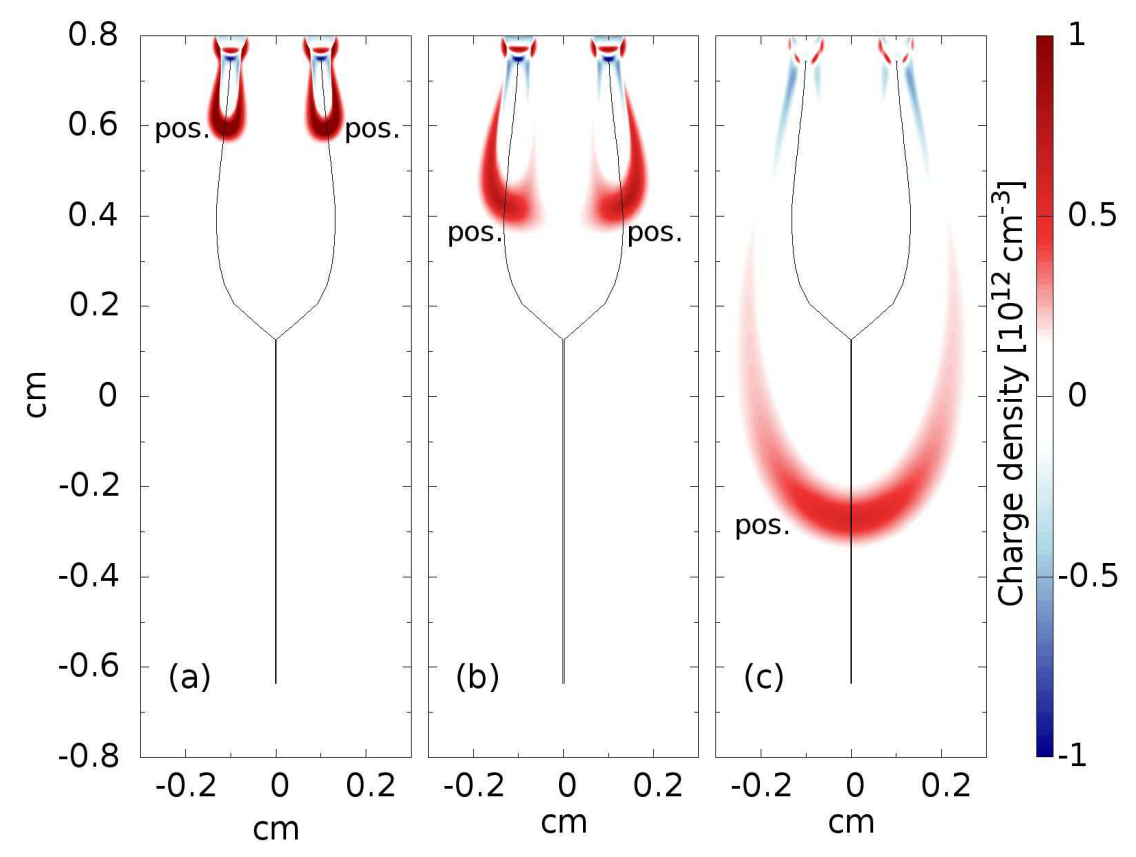

Figure 1: (color online) Time evolution of the net charge density for two positive streamers at ground pressure $\left(N / N_{0}=1.0\right)$ for an applied electric field $E_{\mathrm{a}}=1.5 E_{\mathrm{bd}}$ and two Gaussian seeds with $y_{0}=0.2 N_{0} / N \mathrm{~cm}, n_{\max }=10^{13} N^{2} / N_{0}^{2} \mathrm{~cm}^{-3}$, and $\sigma=0.02 N_{0} / N \mathrm{~cm}$. (a) $t=$ 5.0 ns: well-developed streamers repulsing each other, (b) $t=t_{\mathrm{tr}}=6.3 \mathrm{~ns}$ : transition between repulsion and merging, (c) $t=8.0 \mathrm{~ns}$ : propagation of a single discharge. Black solid line: trajectory of the maximum electric field. 


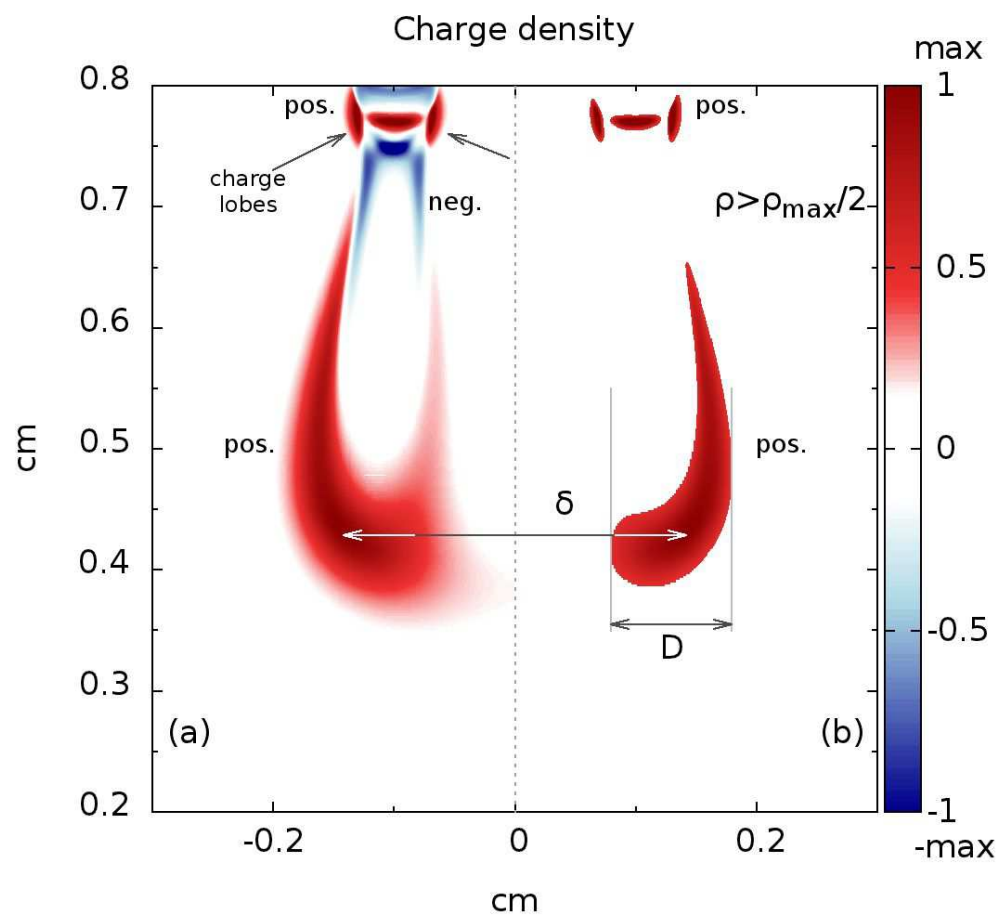

Figure 2: (color online) (a) Net charge density and (b) Region where the net charge density is higher than half the maximum net charge density at $t=t_{\mathrm{tr}}$ for the same condition as in Figure 1(b). Definition of the streamer head characteristic width $\mathcal{D}$ and the mutual distance of both filaments $\delta$. 
ratio $\mathcal{D} / \delta$ increases and reaches a value of 0.4 at the moment of transition ( $4 \mathrm{~ns}$ in case of $y_{0}=0.15 N_{0} / N \mathrm{~cm}$, and $5 \mathrm{~ns}$ in case of $y_{0}=0.20 N_{0} / N \mathrm{~cm}$ ) between streamer repulsion and the beginning of their merging, and streamer merging is then observed. It is interesting to note that when photoionization is omitted, the ratio $\mathcal{D} / \delta$ is less than 0.4 , and no merging is evidenced. Conversely photoionization must be included for positive streamers as it is necessary for their propagation. In this case Figure 3 shows that the ratio $\mathcal{D} / \delta$ increases and reaches a value of 0.35 at the transition time $5.6 \mathrm{~ns}$ for $y_{0}=0.15 N_{0} / N \mathrm{~cm}$, $(6.3 \mathrm{~ns}$ for $y_{0}=0.20 N_{0} / N \mathrm{~cm}$ ), and then streamer merging is observed. These results clearly show the crucial role of the photoionization source term on streamer merging. In the following we show that the value of the ratio $\mathcal{D} / \delta$ at the transition time depends on the polarity of discharges but is practically independent on the multivariable physical settings. For instance, for the same parameters of the seeds as in Figure $1\left(n_{\max }=10^{13} N^{2} / N_{0}^{2} \mathrm{~cm}^{-3}, y_{0}=0.2 N_{0} / N \mathrm{~cm}\right.$, and $\sigma=0.02 N_{0} / N \mathrm{~cm}$ ) we have performed a set of simulations with an applied electric field $E_{\mathrm{a}}$ varying from $1.5 E_{\mathrm{bd}}$ to $2.5 E_{\mathrm{bd}}$ at ground pressure. Figure 4 shows that the resulting values of $\mathcal{D} / \delta$ at the transition times for merging streamers are roughly constant and of about 0.35 for positive polarity discharges, and approximatively 0.4 for negative ones. Hence $\mathcal{D} / \delta$ is independent of the applied electric field $E_{\mathrm{a}}$ for both streamer polarities. Similarly, Figure 5 shows the dependence of $\mathcal{D} / \delta$ on $n_{\max }$, the maximum density of seeds, for a fixed applied electric field $E_{\mathrm{a}}=1.5 E_{\mathrm{bd}}$. Same previous ratios are obtained whereas $\mathcal{D} / \delta$ is slightly decreasing as $n_{\max }$ decreases for positive streamers. However a variation of four orders of magnitude for $n_{\max }$ involves only a variation of about $15 \%$ of $\mathcal{D} / \delta$. In the same way we have tested the variation of $\mathcal{D} / \delta$ with altitude (not shown here). We have considered altitudes ranging from the ground up to $80 \mathrm{~km}$, corresponding to pressure range from 1 to $1.5 \times 10^{-5}$ atm. We have found that the ratio $\mathcal{D} / \delta$ is independent of the altitude and then of pressure in the range studied.

Moreover we have performed simulations to study the influence of the initial seed separation $y_{0}$ on $\mathcal{D} / \delta$. Figure 6 shows the dependence of $\mathcal{D} / \delta$ for $y_{0} \leq 0.2 N_{0} / N \mathrm{~cm}$. Two sets of seed parameters were considered: (a) for $y_{0}>0.1 N_{0} / N \mathrm{~cm}$ : $n_{\max }=10^{13} N^{2} / N_{0}^{2} \mathrm{~cm}$, $\sigma=0.02 N_{0} / N \mathrm{~cm}$, and $E=1.5 E_{\mathrm{bd}}$; (b) for $y_{0}<0.1 N_{0} / N \mathrm{~cm}: n_{\max }=10^{10} N^{2} / N_{0}^{2} \mathrm{~cm}$, $\sigma=0.00736 N_{0} / N \mathrm{~cm}$, and $E=2.5 E_{\mathrm{bd}}$. The first set corresponds to the reference condition used for Figure 1. The second set was chosen to be close to the conditions used in [9], and is characterized by a high value of the applied electric field, comparable to typical values of the electric field in streamer heads, and small initial seed width and separation. Because of the small seed width of the second set, for this set, computations were performed for a space resolution of $1.95 \mu \mathrm{m}$. Figure [6 shows that for positive streamers with $y_{0} \geq 0.04 N_{0} / N \mathrm{~cm}$, the value of the ratio $\mathcal{D} / \delta$ for the second set is slightly less than the reference value of 0.35 for the first set, which can be explained by the small dependence of $\mathcal{D} / \delta$ on $n_{\text {max }}$, as seen in Figure 5. For negative streamers, Figure 6 shows that for $y_{0} \geq 0.04 N_{0} / N \mathrm{~cm}$, the ratio $\mathcal{D} / \delta$ is independent of the value of $y_{0}$. For initial separation of streamers smaller than $y_{0}<0.04 N_{0} / N \mathrm{~cm}$ the transition between repulsion and merging is occurring very close to the initial Gaussian seeds and therefore the region where $\rho \geq \rho_{\max } / 2$ is indistinguishably joined with the charge lobes, typically observed near the initial germs (shown in Figure 2). In this case, the streamer head appears to be significantly wider, and consequently the ratio $\mathcal{D} / \delta$ increases as shown in Figure 6. It is interesting to note that in the two sets used for Figure 6. very different values of $\sigma$ have been used. We have carried out additional simulations to verify that the value of $\sigma$ has a negligible influence on the ratio $\mathcal{D} / \delta$.

Finally, for simulations presented in this work, we have found that a stable value for the ratio $\mathcal{D} / \delta$ is obtained for merging streamers for an initial separation $y_{0}$ which is smaller or comparable to the longest absorption length of photoionization for air $\left(0.1408 N_{0} / N \mathrm{~cm}\right)$. These results clearly show the significant role of photoionization on streamer merging. We 


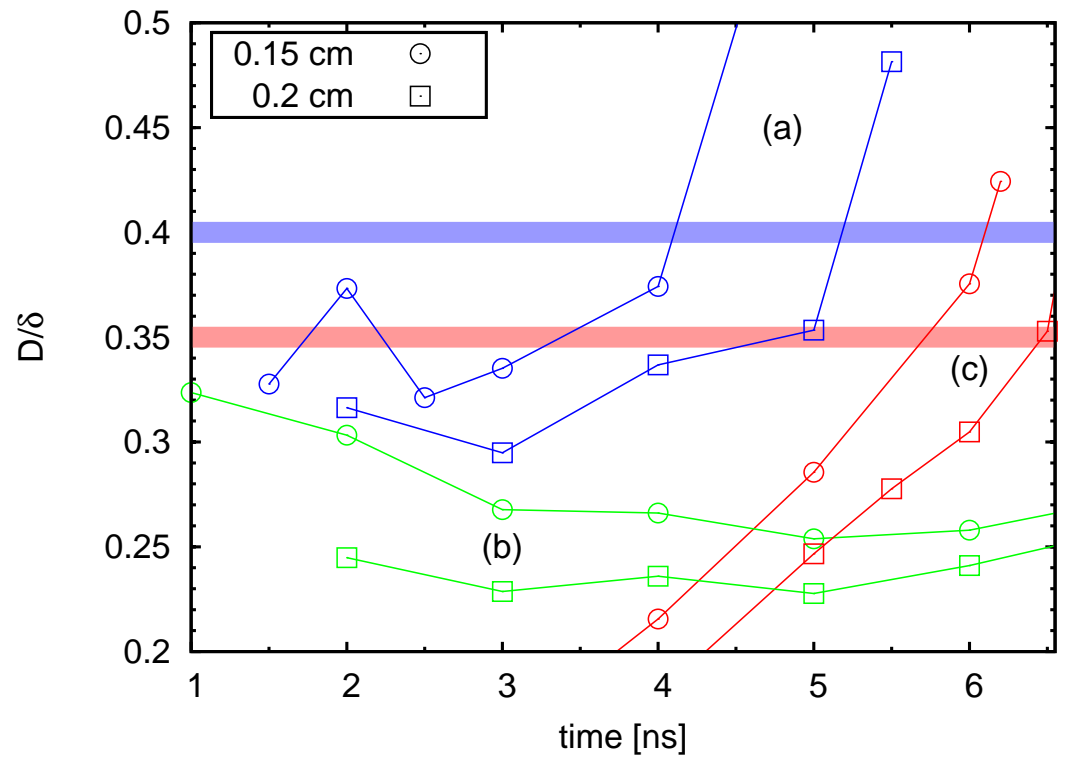

Figure 3: (color online) Time evolution of the ratio $\mathcal{D} / \delta$ for (a) two negative streamers with photoionization source term, (b) two negative streamers without photoionization source term, (c) two positive streamers with photoionization source term. Results for initial separation of the seeds $y_{0}=0.15 N_{0} / N \mathrm{~cm}$ and $y_{0}=0.2 N_{0} / N \mathrm{~cm}$ are shown. Other parameters are similar to Figure 1. Critical merging ratios are indicated with stripes.

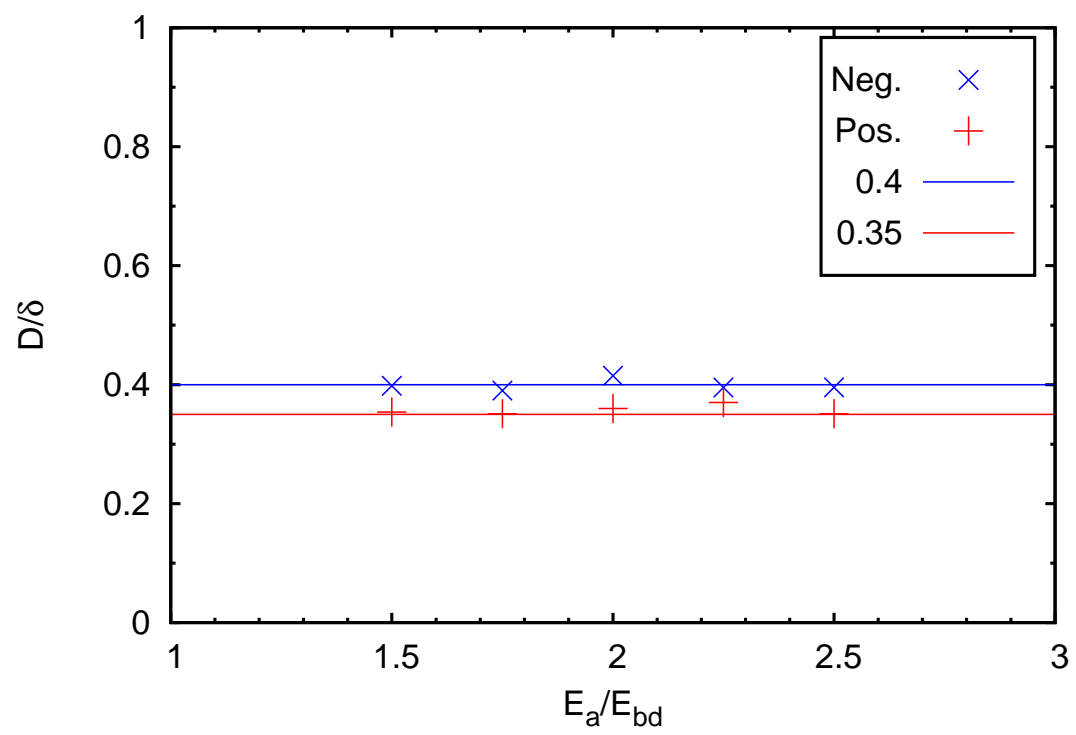

Figure 4: (color online) Ratios $\mathcal{D} / \delta$ for positive and negative streamers for different values of the applied electric field $E_{\mathrm{a}} / E_{\mathrm{bd}}$. Other parameters are similar to Figure 1. 


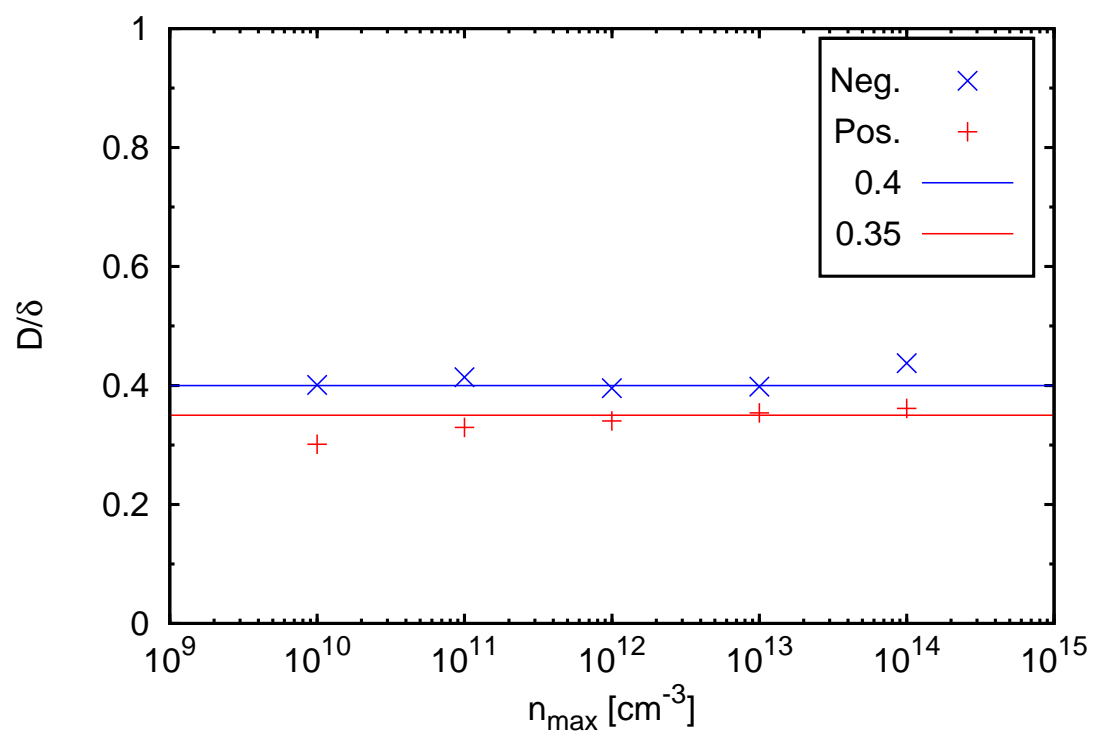

Figure 5: (color online) Ratios $\mathcal{D} / \delta$ for positive and negative streamers for different values of the maximum density $n_{\max }$ of seeds. Other parameters are similar to Figure 1 .

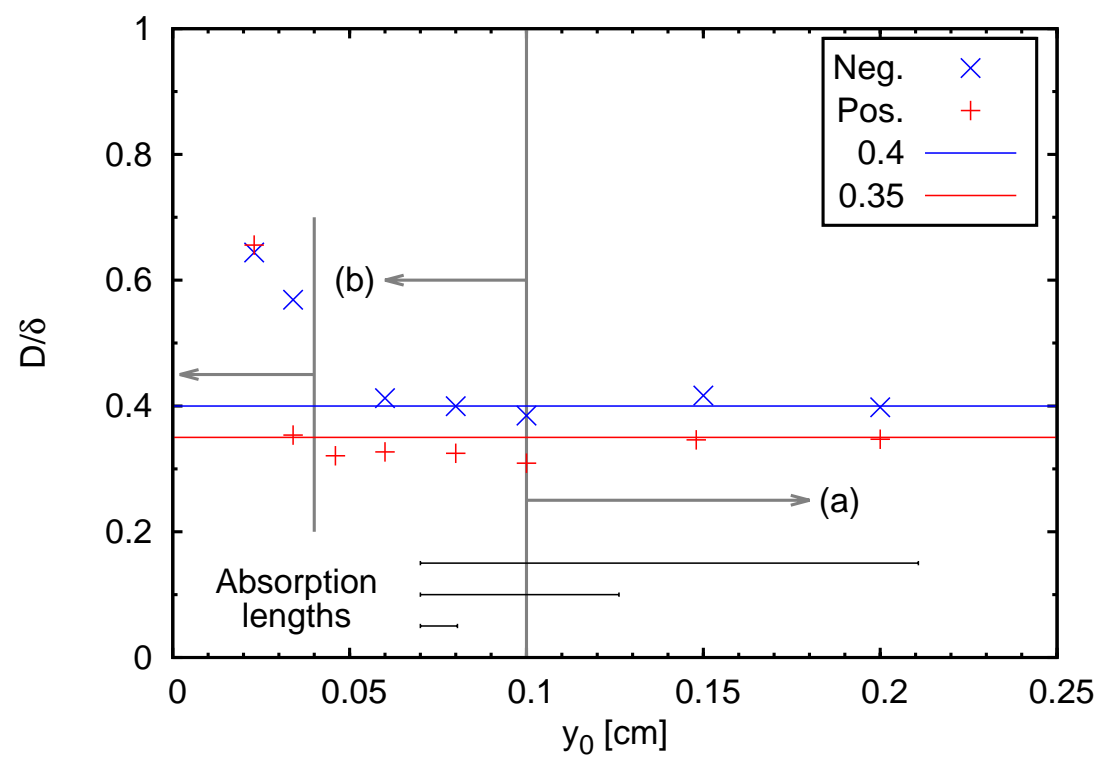

Figure 6: (color online) Ratios $\mathcal{D} / \delta$ for positive and negative streamers for different initial seed distances $y_{0}$, with (a) $n_{\max }=10^{13} N^{2} / N_{0}^{2} \mathrm{~cm}, E=1.5 E_{\mathrm{bd}}, \sigma=0.02 N_{0} / N \mathrm{~cm}$; (b) $n_{\max }=$ $10^{10} N^{2} / N_{0}^{2} \mathrm{~cm}, E=2.5 E_{\mathrm{bd}}, \sigma=0.00736 N_{0} / N \mathrm{~cm}$. Absorption lengths for photoionization at ground pressure are shown. 
have carried out simulations for larger values of $y_{0}$, but it was not possible to derive simple conclusions on streamer interactions from the analysis of the value of the ratio $\mathcal{D} / \delta$.

In conclusion, in this work, we have carried out a parametric study on the interaction of two streamers in air using 2D numerical simulations based on an accurate numerical method which ensures a time-space error control of the solution. This study is a first step towards a quantitative analysis of streamer merging in real 3D conditions. We have shown that for a initial separation between streamers that is smaller or comparable to the longest characteristic absorption length of photoionization $\left(0.1408 N_{0} / N \mathrm{~cm}\right)$, streamers will start to merge, i.e., the distance between their trajectories starts to decrease despite their electrostatic repulsion, at the moment when the ratio $\mathcal{D} / \delta$ of the streamer characteristic width $\mathcal{D}$ and the streamer mutual distance $\delta$ attains a value of about 0.35 for positive streamers, and 0.4 for negative ones. These ratios are independent of the applied field, the initial seed configuration, and pressure. Moreover presented results clearly illustrate the significant role of photoionization on the streamer merging. Further studies are required to find the key parameters to characterize streamer interactions when $y_{0}$ is considerably larger than the longest photoionization absorption length.

\section{Acknowledgements}

This research was supported by a fundamental project grant from ANR (French National Research Agency): Séchelles (ANR-09-BLAN-0075-01, PI. S. Descombes), and by a DIGITEO RTRA project: $M U S E$ (PI. M. M.). Z. B. acknowledges support by project CZ.1.05/2.1.00/03.0086 funded by European Regional Development Fund and support of Ecole Centrale Paris.

\section{References}

[1] Raizer Y P 1991 Gas Discharge Physics (Springer-Verlag, Berlin Heidelberg, 1991)

[2] Sentman D D, Wescott E M, Osborne D L, Hampton D L, and Heavner M J 1995 Geophys. Res. Lett. 22 1205-8

[3] Pasko V P 2007 Plasma Sources Sci. Technol. 16 S13-S29

[4] Briels T M P, Kos J, van Veldhuizen E M and Ebert U 2006 J. Phys. D: Appl. Phys. $395201-10$

[5] Cummer S A, Jaugey N, Li J, Lyons W A, Nelson T E and Gerken E A 2006 Geophys. Res. Lett. 33 L04104

[6] Nijdam S, Geurts C G C, van Veldhuizen E M and Ebert U 2009 J. Phys. D: Appl. Phys. 42045201

[7] Naidis G V 1996 J. Phys. D: Appl. Phys. 29 779-83

[8] Luque A, Brau F and Ebert U 2008 Phys. Rev. E 78016206

[9] Luque A, Ebert U and Hundsdorfer W 2008 Phys. Rev. Lett. 101075005

[10] Brau F, Luque A, Meulenbroek B, Ebert U, and Schäfer L 2008 Phys. Rev. E 77026219

[11] Babaeva N Y and Naidis G V 1996 J. Phys. D: Appl. Phys. 29 2423-31

[12] Kulikovsky A A 1998 Phys. Rev. E 57 7066-74 
[13] Bourdon A, Pasko V P, Liu N Y, Celestin S, Segur P and Marode E 2007 Plasma Sources Sci. Technol. $163656-78$

[14] Liu N Y, Celestin S, Bourdon A, Pasko V P, Segur P and Marode E 2007 Appl. Phys. Lett. 9121211501

[15] Duarte M, Bonaventura Z, Massot M, Bourdon A, Descombes S and Dumont T $2012 \mathrm{~J}$. Comput. Phys. 2313 1002-19

[16] Amestoy P R, Duff I S, Koster J and L'Excellent J-Y 2001 SIAM J. Matrix Anal. Appl. $23115-41$

[17] Amestoy P R, Guermouche A, L'Excellent J-Y and Pralet S 2006 Parallel Computing $322136-56$

[18] Liu N and Pasko V P 2006 J. Phys. D: Appl. Phys. 39 327-34 